\title{
Modeling Real-Time 3-D Lung Deformations for Medical Visualization
}

\author{
Anand P. Santhanam, Celina Imielinska, Paul Davenport, Patrick Kupelian, and Jannick P. Rolland, Member, IEEE
}

\begin{abstract}
In this paper, we propose a physics-based and physiology-based approach for modeling real-time deformations of 3-D high-resolution polygonal lung models obtained from highresolution computed tomography (HRCT) images of normal human subjects. The physics-based deformation operator is nonsymmetric, which accounts for the heterogeneous elastic properties of the lung tissue and spatial-dynamic flow properties of the air. An iterative approach is used to estimate the deformation with the deformation operator initialized based on the regional alveolar expandability, a key physiology-based parameter. The force applied on each surface node is based on the airflow pattern inside the lungs, which is known to be based on the orientation of the human subject. The validation of lung dynamics is done by resimulating the lung deformation and comparing it with HRCT data and computing force applied on each node derived from a 4-D HRCT dataset of a normal human subject using the proposed deformation operator and verifying its gradient with the orientation.
\end{abstract}

Index Terms-Green's function, lung physiology, organ morphology.

\section{INTRODUCTION}

$\mathbf{M}$ EDICAL modeling, simulation, and visualization constitute a framework for the development of application tools for teaching and training in medicine. When coupled with a stereoscopic visualization paradigm such as virtual and augmented reality (V/AR), 3-D visualization is enabled. Simulated physiological lung models may facilitate a better understanding of lung physiology for the trainees [1]. Clinicians may utilize such models, derived from a patient-specific data, to determine immediate treatment course and future interventions for the patient. The need for such visualization arises from the cost and complexity in capturing temporal continuous patient-specific lung dynamics within a single breathing cycle.

Deformation methods can capture the shape change in a 3-D breathing lung model, a key step in modeling of lung dynam-

Manuscript received February 20, 2006; revised April 24, 2006. This work was supported by the U.S. Army STRICOM and by the Florida Photonics Center of Excellence.

A. P. Santhanam is with the M.D. Anderson Cancer Center Orlando and the College of Optics and Photonics, University of Central Florida, Orlando, FL 32816 USA (e-mail: anand@odalab.ucf.edu).

C. Imielinska is with the Department of Biomedical Informatics, Columbia University, New York, NY 10027 USA (e-mail: ci42@columbia.edu).

P. Davenport is with the Department of Physiological Sciences, University of Florida, Gainesville, FL 32611 USA (e-mail: pdavenport@vetmed.ufl.edu).

P. Kupelian is with the Department of Radiation Oncology, MD Anderson Cancer Center, Orlando, FL 32806 USA (e-mail: Patrick.kupelian@orhs.org).

J.P. Rolland is with the College of Optics and Photonics, School of Computer Science and also with the Institute of Simulation and Training, University of Central Florida, Orlando, FL 32816 USA (e-mail: jannick@odalab.ucf.edu).

Color versions of one or more of the figures in this paper are available online at http://ieeexplore.ieee.org.

Digital Object Identifier 10.1109/TITB.2007.899489

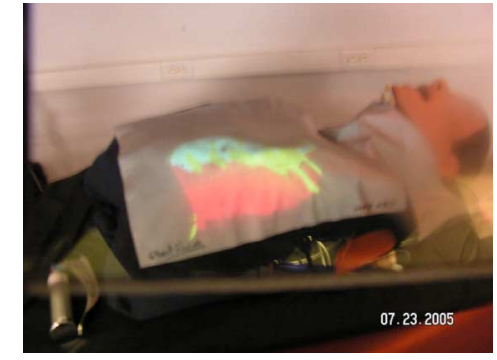

Fig. 1. Visualization of nondeforming lungs when registered with a human patient simulator.

ics. Such methods facilitate accurate deformation of a 3-D lung model from its shape at the beginning and at the end of the inhalation cycle. Of particular importance is modeling the shape change of high-resolution 3-D models with a large number of elements (e.g. nodes, triangles) [1]. Such high-resolution lung models may be obtained from either the computed tomography (CT) imaging and controlled mechanical ventilation [2], or from the visible human data [1]. The large number of elements in these high-resolution 3-D models contributes to computational complexity of the deformation computation and graphical rendering; therefore, limiting the real-time capabilities of the application.

The real-time requirements of the deformation algorithm may be best understood in the context of a general description of the AR environment employed in the 3-D visualization as previously described in [3]. The components of this AR environment consist of a head-worn display (HWD), an optical tracker, a few landmarks in the physical world, and a virtual scene to be rendered. The landmarks are placed on a physical human patient simulator. The optical tracker tracks the position of the landmarks and the position and orientation of the HWD at the rate of once per $15 \mathrm{~ms}$. A virtual static 3-D lung model is superimposed and viewed through the HWD on the human patient simulator. Any changes in the position and orientation of the HWD are tracked and the superimposed virtual lung model is updated and rendered according to the new viewpoint. Fig. 1 shows static lungs superimposed over the human patient simulator. To replace this static model with physiologically correct deformable lung model that is displayed without flicker, the deformation must be computed at greater than one frame per $15 \mathrm{~ms}$. In order to achieve this challenging rate of deformation, we present a physically based deformation method that is combined with pre-computation.

This paper is structured as follows. Section II summarizes the related work on physically based deformation algorithms, tissue deformation methods, and lung deformation 
methods, Section III discusses the proposed deformation method, Section IV discusses the validation of 3-D lung dynamics using 4-D high-resolution computed tomography (HRCT) datasets, and Section V discusses the real-time deformations.

\section{RELATED WORK}

In this section, we overview related work on physically based deformation methods and discuss tissue and lung deformation methods.

\section{A. Physically Based Deformations}

Physically based deformation methods use a differential equation-based formulation that allows modeling deformations under dynamic conditions. These deformation methods can be used extensively in areas ranging from basic animations to surgical simulations. They can, however, be classified into two groups, which are: 1) dynamic and 2) static. While the dynamic methods more precisely represent the transient stages of the deformation, the static methods compute the equilibrium or final values of deformation.

A brief review of these deformation methods is as follows. Initial models for dynamic physically based deformations were pioneered for modeling rigid-body simulation [4]. While Platt used mass-spring models for creating animated facial expressions [5], Terzopolous used finite-difference methods (FDMs) to create simple animations [4]. Of particular interest is the mass-spring model that has been widely used in modeling deformations [6]. Specific implementations of mass-spring models can be seen for cloth simulations [7], facial animations [8], human animations [9], and organ deformations [10]. Additionally, the mass-spring model was modified into a mass-springdamper model, which was suitable for modeling flow-induced vibrations and was discussed by Blevins [11]. These methods represent the mechanics of deformation as a second-order differential equation of the position change for a vertex on the 3-D model. The FDM was further modified to simulate deformation of rigid bodies that lead to its fracture. These formulations were computationally expensive for high-resolution models. The methods that improved computational complexity were based on either decimating the 3-D model or simplifying the equation complexity. The first approach used graph simplification algorithms [12], while the second approach used matrix simplification methods [13]. An integration of both approaches was done using multiresolution wavelets [14]. However, the performance of these dynamic deformation methods is limited by the real-time requirements of the computing system. Additionally, an increase in the number of elements of the 3-D models increases the graphical rendering complexity.

To achieve real-time deformation, a linearization of dynamic deformations may be used. This linearization can be implemented using a first-order differential representation of equation of the motion. Generally, the linearity in deformations occurs only for smaller and nontorsional deformations [15]. A key advantage of linearization of dynamic deformations is the possibility to precompute the deformation. Once the deformation is precomputed for a unit force, the deformation is linearly com- puted according to the applied force [16]. Iterative solutions for solving the first-order differential equation for precomputation purposes have also been proposed for structural modeling purposes [17].

Static physically based (elastostatic) methods represent linearized dynamic methods that further improve the real-time capabilities by directly computing the equilibrium or the final position of a 3-D model when a force is applied. First static methods were introduced by Lord Kelvin in 1848 for bending an elastic rod. Furthermore, Lagrange suggested a tensor-based method for computing the equilibrium displacements of the elastic rod [18]. This method was further improved by Green and was referred to as Green's function [19]. The Green's matrix or tensor mathematically relates the displacement of a node to the force applied on the node and its neighboring nodes, for a given 3-D mesh. A measurement of Green's matrix for a given 3-D real object was done by computing the aforementioned deformation for an applied force on a node. The surface properties of the 3-D mesh were taken to be homogenous (i.e., surface has the same material properties at all the surface points), which renders the computation to be inexpensive [20].

A fundamental solution to heterogeneous elasticity problems using Green's function was given by Nakamura [21] and Ting [22]. While these methods were used in different applications, they were based on inverting a known Green's tensor [23]. The material properties used in the Green's tensor were the elastic moduli of each surface point [22]. However, a method to represent a heterogeneous Green's matrix in a computationally inexpensive manner still poses a challenge.

\section{B. Physically Based Tissue Deformations}

The methods for representing tissue deformation were discussed in the context of the biomechanical properties of tissues in [24]. They were further developed to provide a basis for defining static deformation methods for tissue modeling [25]. An analysis of soft-tissue modeling using the linearized elastostatic reversible deformation approach was introduced by Delingette [26]. To deform models that represent tissue samples and organs, finite-element methods (FEMs) were used, and the computations were further optimized using condensation techniques [13]. The inability of linearized deformation approaches to model rotational tissue deformations was addressed by Ayache [27]. A nonlinear tensor model based on St. Venant-Kirchoff model was further discussed [27]. Linear element methods [28] based on tensor deformations were introduced in order to model interactive tissue deformations. This method was further modified to include torsional deformations, and was referred to as radial element methods [29]. The scalability and run-time complexity of these methods for deforming high-resolution models need to be addressed further. An approach of particular importance that is based on weak FEM [17] with hierarchical levels of detail for soft-tissue simulation was presented by Hauth [30]. In this method, Green's function was used for representing the strain tensors (ratio of the change in length to the actual length). The stress (force per unit area) tensor was represented as a convolution equation of integration time 
for viscoelastic materials. The transfer matrices were further computed using an iterative approach. The heterogeneous elasticity of the tissue was taken into account for every element by using a negative exponential function of the Lame's constants of its neighboring elements [31].

\section{Lung Deformations}

Lung deformations have been studied for verifying different brands of medical imaging equipments such as myocardial SPECT [32]-[34], understanding pulmonary mechanics, registering MRI images, generating in vitro lung models, and for medical training purposes [35]. The initial methods to model the 3-D human lung deformation were based on physiology and clinical measurements [36]. Significant amount of work has been undergone in understanding and modeling pulmonary mechanics using animal and human data. Key parameters extracted from pulmonary imaging modalities are the Green's strain tensor and Jacobian of the displacement gradient. While the Lagrangian strain tensor provided the change in length of the edges in the 3-D data, the Jacobian of the displacement gradient provided insights on the local change in lung volume.

From a simulation and visualization perspective, we concentrate on deforming a given 3-D human lung model for a known airflow inside it. It is done by using both the stress and strain components at every lung node, and using a physics-based deformation paradigm that relates the stress and strain in a local lung neighborhood. Under this context, the human lung modeling literature has been mainly divided into two approaches, which are: 1) single-compartment model and 2) multicompartment model. The physically based deformation of the human lung model as a linearized single-compartment model was proposed by Promayon [37]. An FEM-based single-compartment model was proposed by Decarlo for real-time medical visualization [38]. It was then extended by Kaye in order to model pneumothorax related conditions [39]. Additionally, a visualizationbased training method was developed for pneumothorax using a single-compartment model [35]. The method had an analogy for lung deformations to an electrical circuit [39]. Another FEMbased single-compartmental surface lung model was developed from HRCT data of patients with lung tumors. Using this FEM representation, CT images were simulated at different breathing phases and used for preoperative treatment planning. Such procedures not only modeled the lung surface deformations but also modeled the motion of lung tumors inside the lung during breathing. The lung surface and lung tumor motions were validated using patient data. The real-time issues in this approach were not discussed [40].

A multicompartment functional FEM model, which modeled the tissue constituents (i.e., parenchyma, bronchiole, and alveoli) of lungs was done by Tawhai. This effort aimed in analyzing the anatomical functions of lungs during breathing [41]. The run-time computational complexity of this approach was reduced by modeling, solely, the bronchioles and the airflow inside the lung [42]. Of particular importance is the role of airflow inside lungs. Based on the medical image analysis, the spatial air distribution inside lungs was shown to be dependent on the gravity, and thus, the orientation of the subject. From the perspective of a physically based deformation, the air distribution defines the force applied on the lung model, and thus, needs to be accounted for.

A nonphysically based analysis of lung morphology has been extensively investigated in the field of lung physiology and imaging. Some of the key works include the analysis of lung morphology using image warping [43]. A nonphysically based method to lung deformations was also proposed using nonuniform rational B-splines (NURBS) surfaces based on imaging data from CT scans of actual patients [33]. The usage of a highresolution model for lung deformations and its real-time visualization were not addressed in these efforts. From a modeling and simulation perspective, the physically based deformation methods are apt for simulating lung dynamics since they allow the inclusion of different breathing parameters.

\section{PROPOSED METHOD}

In this section, we present a method for deforming and visualizing 3-D lung dynamics. The proposed model of lung dynamics is based on the analysis of both pulmonary measurements to account for the physiology and CT imaging datasets to account for the morphology. The usage of both single and multicompartmental models for lungs was discussed in Section II-C. Although the multicompartment model for 3-D lung dynamics can be observed to be a more accurate model for 3-D lungs, its visualization cannot be in real time due to the high computational complexity induced by the collision detection among the lobes. The single-compartment model has the ability to satisfy the real-time requirement of the visualization system. Segars and Kaye have also previously validated the accuracy of the single-compartment model. Since our aim is to develop 3-D real-time deformable lung models that can be integrated with the AR-based medical visualization systems, we choose a singlecompartment model for representing the 3-D lung dynamics. Two components that are addressed in the proposed method of 3-D lung dynamics are: 1) pressure-volume (PV) relationship of lungs and 2) physics- and physiology-based deformation of lungs. The former is introduced and discussed briefly in Sections III-A and B. Specifically, Section III-A presents the physiology of breathing, where the sequence of steps in the respiratory process that leads to the inhalation and exhalation is described. The role of the PV relation in representing this physiology is discussed. Section III-B presents a discussion on the pulmonary measurement and parameterization of the PV relation. The physics- and physiology-based deformation of lungs that forms the main topic of the paper is detailed in Sections IIIC-F. Specifically, an introduction of the proposed deformation is given in Section III-C. Section III-D presents a discussion on the modifications done to the 3-D polygonal lung model, in order for the model to be deformable. The mathematical model of the deformation method is discussed in Sections III-E and F.

\section{A. Physiology of Breathing}

The first step in the respiratory process is initiated by the anatomical components that deal with the control of ventilation. 
The control of ventilation is managed at the highest level by a network of neurons that starts with the brainstem and ends with spiral motor neurons. Such networks of neurons drive the muscles of breathing. The motor drive of breathing controls the respiratory rhythm and the adaptation to physical conditions. The respiratory rhythm is generated by the central respiratory pattern generator (CRPG), which is formed by synaptic connections of neurons generating a specific firing pattern in the brainstem. The outputs of the CRPG are the phrenic activation and intercostals activation. While the phrenic activation controls the diaphragm movement, the intercostal activation controls the intercostal muscles movement. These activations innervate the muscles of the thoracic cavity, and the motor units of the respective muscles respond. The diaphragm is the muscle that forms the floor of the thoracic cavity. It is made of two sets of fibres, namely, costal (anterior part of the diaphragm) and crural (posterior part of the diaphragm). The diaphragm contracts during the inspiration cycle and relaxes during the expiration cycle, creating an upwarddownward (cranio-caudal) movement. The intercostal muscles mainly constitute the rib cage, and have a forward-backward (anterior-posterior movement). The combined output of the activations causes the nonuniform change in shape of the thoracic cavity, which subsequently leads to the decompression of air inside the lungs and a decrease in pressure inside the lungs. This decrease in pressure leads to the flow of air inside lungs. The relation between the change in pressure and volume is referred to as a PV relation. The PV relation reflects key respiratory parameters of the patient such as lung tissue properties and also indicates the presence of changes in pulmonary mechanics. The ventilation and the movement of air are dependent on the compliance of the airway and tissue resistance of the lungs during breathing. The regional change in 3-D lung shape was observed by [44] to be mainly attributed to the change in the dimensions of alveolus and alveolar ducts. This change can be related to the change in the alveolar blood pressure and alveolar air volume. The blood pressure in alveolus and alveolar ducts was discussed by [44] to be a constant throughout the breathing process. Thus, the regional change in lung shape depends on the alveolar air volume capacity. From a modeling and simulation perspective, the two key components related to the physiology that are to be taken into account are the PV relation that provides the details on the volume of airflow inside lungs and the alveolar expandability (alveolus and alveolar duct expandability) that provides the regional air distribution inside the lungs.

\section{B. Extraction and Parameterization of PV Datasets}

A method to parameterize the PV relation is discussed in [45] and [46]. From a modeling and simulation perspective, such an approach allows us to model the PV relations of both normal and disease states (e.g., chronic obstructive pulmonary disease and dyspnea). The method takes into account both the control of ventilation and the muscle mechanics. Such an approach allows us to simulate PV curves in different breathing conditions, which can then drive the simulation of 3-D lung dynamics for medical visualization applications. The PV relation was represented using both a second-order differential equation that represents the increase and decrease in volume, and a nonlinear control function that represents the summary muscle resistance. The control function was given as a linear summation of products of control parameters and a set of basis functions. The basis functions allowed us to steer the control function that accounts for variations in the breathing condition. The proposed method can be reversed in order to estimate the values of control parameters from human subject data. Results show that a set of five control parameters may define accurately the PV relation system. The associated PV relation showed less than 1\% RMS difference with the normal human subject data.

The validation of the proposed PV relation using pulmonary measurements from human subjects was done as follows. A set of two adult subjects was considered. They were informed of the purpose of the study prior to starting the experiment and consent was obtained. The Institutional Review Board at the University of Florida reviewed and approved this project. Two adult men with no history of pulmonary or cardiovascular disease participated in this study. At the beginning of the experiment, a standard set of instructions was presented to each subject, informing them of their task. Each subject was told to respire as normally as possible. The subjects were seated comfortably in a chair. The subject wore a nose clip and breathed through a mouthpiece connected to a nonrebreathing valve. Care was taken to suspend the valve to eliminate the need for the subjects to bite the mouthpiece yet maintain an airtight seal. The pressure at mouth (PM) was recorded from a port in the center of the valve. In esophageal pressure, Pes was recorded. A thin-walled latex balloon (length of $10 \mathrm{~cm}$ and a diameter of $3.5 \mathrm{~cm}$ ) was placed over a polyethylene catheter $(\mathrm{i} . \mathrm{d}=0.14 \mathrm{~cm})$. The balloon catheter was connected to a calibrated differential pressure transducer (Micro Switch, 14 PC). A topical anaesthetic (Citacaine 2\%) was applied to the oropharynx before each experiment to reduce the gag reflex, and the balloon was lubricated with $2 \%$ viscous xylocaine. Pes was measured by advancing the balloon catheter transnasally down the esophagus until the balloon was in the middle third of the esophagus. During calibration, Pes sensitivity was adjusted to 0 . The subjects were also asked to close their eyes throughout the experiment to prevent eye blinks and reduce visual distractions. The subjects inspired through the nonrebreathing valve with a pneumotachograph placed between the mouthpiece and the mouth port of the valve. The pneumotachograph was connected to a differential pressure transducer for the measurement of airflow $\mathrm{V}^{\prime}$. The volume was measured by electrical integration of the airflow signal. PM, Pes, $\mathrm{V}^{\prime}$, and $\mathrm{V}$ were digitalized and stored on computer disc for subsequent analysis. Pes-V curves were obtained for quiet breathing and a slow vital capacity maneuver for each subject. The cardiac artifact was removed by digital filtering of the Pes signal. Fig. 2(a) and 2(b) show the normalized PV curves extracted from the human subjects and the normalized PV curves parameterized for each of the subject data. The figures show outstanding agreement with the subject data and their parameterization.

\section{Lung Morphology}

-D lung deformations can be effectively understood by analyzing the 3-D lung morphology obtained from CT imaging 


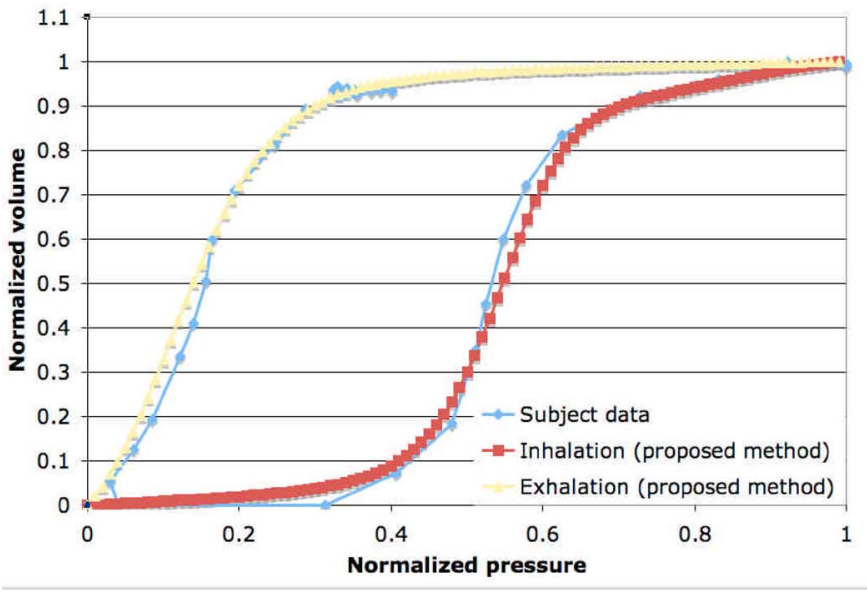

(a)

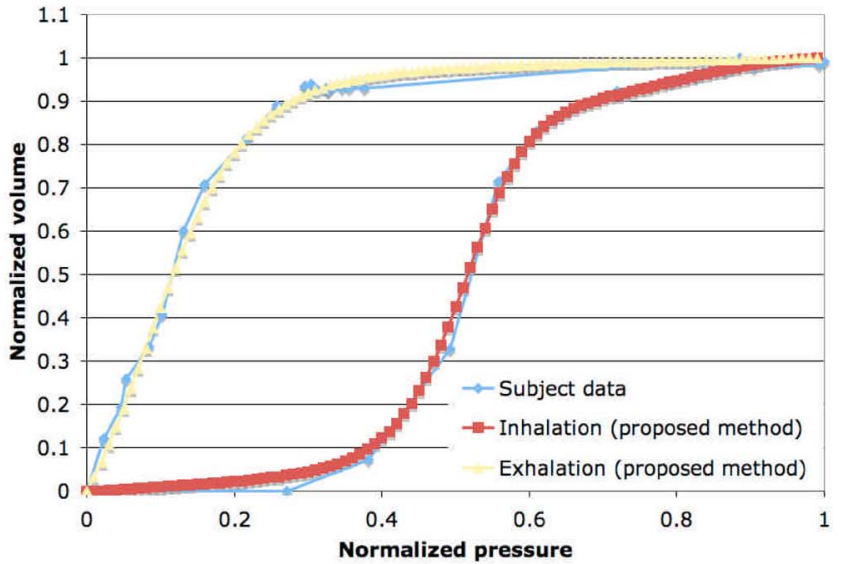

(b)

Fig. 2. (a) and (b) PV curves measured from two human subjects and their parameterized representation.

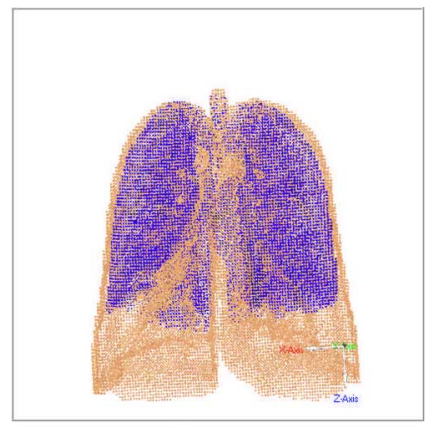

(a)

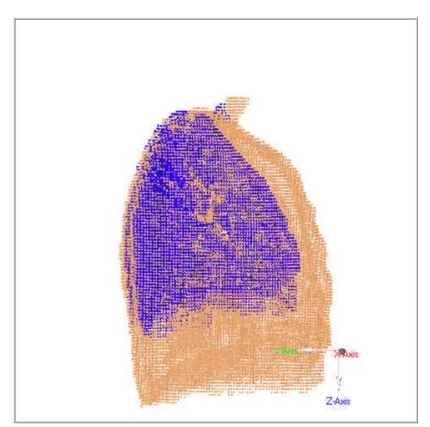

(b)
Fig. 3. (a) Frontal view of human 3-D lung model at the start of inhalation (dark gray) and at the end of inhalation (light gray). (b) Side view of human 3-D lung model at the start of inhalation (dark gray) and at the end of inhalation (light gray).

scans. The datasets have been collected as follows. Four CT scans of a normal subject lying in the supine position were obtained by Dr. E. A. Hoffman from the University of Iowa [47]. Each scan was taken at a different time point during respiration with the lung volume held at approximately 5, 40, 75, and $100 \%$ of the vital capacity. It was measured by a pneumotachometer, and a high-frequency balloon valve prohibited the airflow at the mouth when the lung volume reached the desired level on the expiratory limb [2]. The surfaces for the right and left lungs were computed from each scan and converted into 3-D mesh models by using the segmentation functions from the Analyze software [48].

Fig. 3(a) and (b) show the frontal and side view of the 3-D lung models obtained in a supine position at the start of the inhalation (dark gray model) and at the end of the inhalation (light gray model) cycle. In Fig. 3(a), the cranio-caudal displacement of the lung base is shown to be greater than that of the lung apex. In Fig. 3(b), the anterior-posterior displacement of the apex region of the lungs, which is minimal as compared to the base region of the lungs, is showed. Such nonuniform changes in lung dimension is caused by: 1) the nonuniform change in the thoracic cavity dimension; 2) the heterogeneity of the lung tissue elasticity; and 3) the regional variation in the airflow caused by the gravity. From the lung physiology, it can be seen that the change in thoracic cavity dimensions is caused by the contraction of the diaphragm and the rotation of rib cage during breathing, both of which can vary for everyone. In Fig. 3(a) and (b), the base of the lungs shows no significant variations in the costal and crural diaphragm segments, and thus, the image data show the uniform movements of the diaphragm segments. In this paper, we thus consider the rate of change of costal and crural segments to be 0 , as seen in the CT images, which allows us to focus on modeling the shape change induced by lung tissues.

It can also be observed from clinical data that there exists linearity in the change in lung dimensions with an increase in volume. Fig. 4(a) represents the linear change in the dimensions of the lung's bounding box (a box that exactly encloses the 3-D lungs) with an increase in volume during a single breathing cycle. Fig. 4(b) represents the linear change in the ratio of the lung volume and the bounding box volume as the lung volume increases. The ratio is also seen to lie in a close range of $0.32-0.40$.

A clinical analysis of the linearity in individual node displacements was presented in [47]. In summary, a set of landmarks were chosen and tracked in the sequence of 3-D models obtained during a single breathing cycle. The magnitude of the landmark displacements was observed to be linear with an increase in volume. Thus, the change in lung shape can be modeled as a linear function of the change in lung volume. The directional component of the nodal displacement was not reported. In this paper, the directions of the displacements of nodes in the surface of the 3-D lung model are taken as a constant during a single breathing. The constant direction is motivated by two anatomical facts, which are: 1) the radial and linear expansion of alveoli throughout a breathing cycle (based on morphometric analysis) [49] and 2) the pleural liquid that allows the lungs to slide during the rib cage rotation [50]. From a mechanical perspective, the constant direction of nodal displacement is supported by the work minimization principle [51]. This property is further analyzed in Section III-F. 
Change in bounding box of the left and right lungs

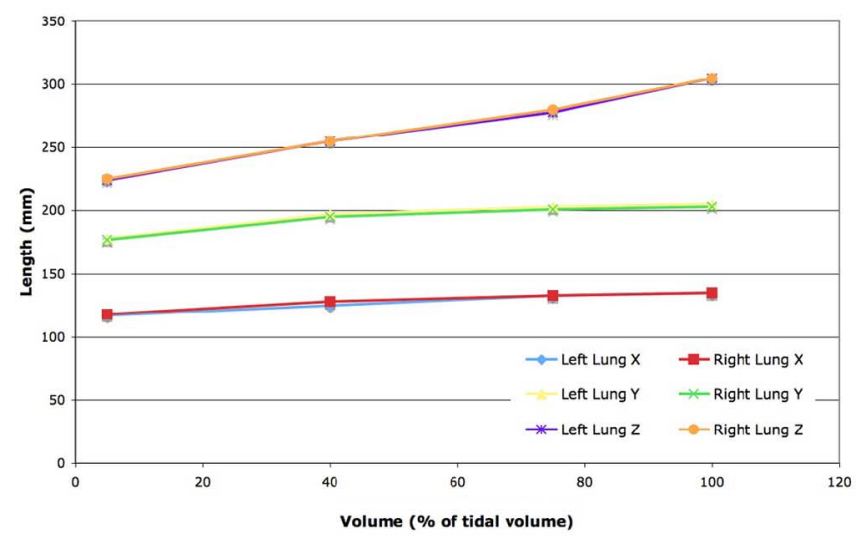

(a)
Ratio of lung volume and the bounding box volume

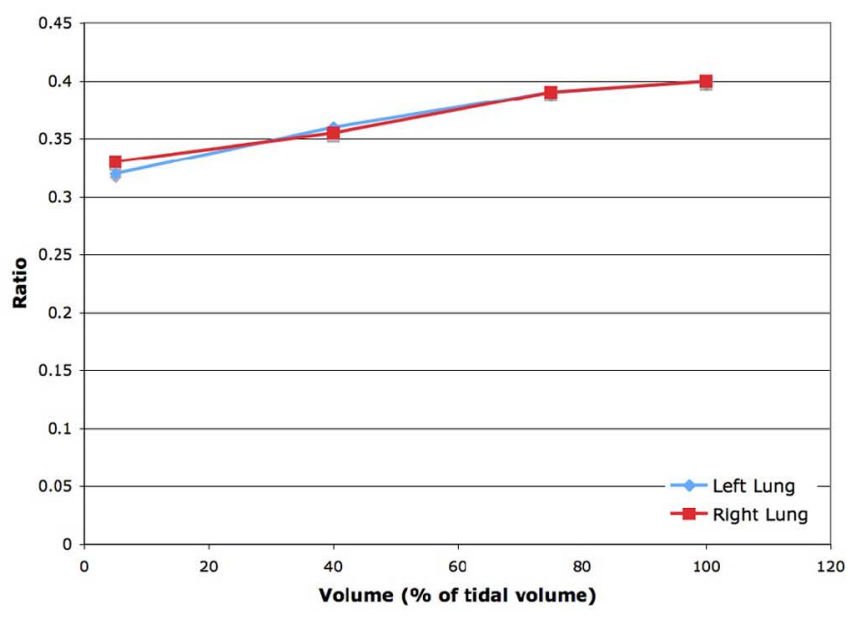

(b)

Fig. 4. (a) Change in the length of the lung's bounding box. (b) Ratio of lung volume to the change in bounding box volume.

\section{Outline of the Proposed Method for Real-Time} 3-D Lung Dynamics

The first component of the proposed methodology was detailed in Section III-A. The second component of the proposed methodology constitutes the main contribution of this paper, which estimates the localized change in the lung shape as a function of the increase in lung volume [52]. The proposed method of visualization can accommodate deformation of a high-resolution 3-D human lung model. The usage of highresolution models is needed in modeling both normal and pathophysical lung dynamics with high fidelity. The change in lung shape is modeled using an elastostatic deformation method. This deformation method has the following properties. First, it compensates for the lack of information about the lung tissue's elastic heterogeneity by using an estimate of local tissue elasticity. Second, the method accounts for the fact that there are no contact forces at surface points, as commonly encountered in physically based deformation methods, given the air flowing everywhere inside the lungs. The method is detailed in Sections III-E-III-G.

The third component of the methodology adopted for the simulation of 3-D lung dynamics deals with the implementation of 3-D lung deformations in a graphics-processing unit, and is summarized in Section V and detailed in [53].

\section{E. 3-D Model Representation}

We now explain the modifications applied to a 3-D lung mesh model, then present the method for computing the magnitude of the node displacement. The input is a high-resolution mesh lung model that is to be deformed using the following modifications. For each link of the polygonal model, we add a node called a spring node. The spring node is used to damp the flow of applied forces between two mass nodes. A schematic representation of the mass-spring model together with the proposed model is shown in Fig. 5(a). Additionally, each node in this modified polygonal model is given an associated attribute rep-
Model Discretization
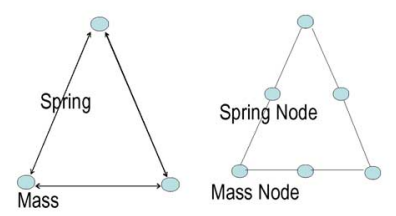

MASS-SPRING MODEL PROPOSED METHOD

(a)

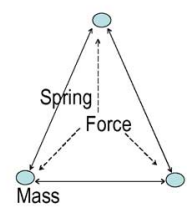

MASS-SPRING MODEL

(b)

Fig. 5. (a) Schematic representation of a discrete 3-D model using the proposed approach and the corresponding representation using a mass-spring model [5]. (b) Schematic representation of force application in the proposed approach as well as in the mass-spring model.

resenting its stiffness. These stiffness values are equivalent to the Young modulus that represents the ratio of the strain experienced by the node to the stress applied [31]. These values are assigned based on the regional alveolar expansion of the human lungs [50]. In the proposed approach on every mass node, a force is applied. A schematic representation of force application is as shown in Fig. 5(b). There are two ways in which the applied force for a mass node can be set. First, it can be estimated based on the orientation of the lungs since the airflow inside the lungs is caused by the vertical pressure gradient. The computation of displacement of every node from this applied force is discussed in Sections III-F and III-G. Second, the applied force on every node can also be more accurately extracted using clinical data combined with the mathematical model discussed in Section III-F. This extraction of applied force from the clinical data is further explained in Section IV-B.

\section{F. Mathematical Model}

We now explain the mathematical model that is used to compute the displacement of mass nodes for a given applied force. Let the mass, displacement, and velocity of the $i$ th node be $M[i], D[i]$, and $D^{\prime}[i]$, respectively. The velocity (using 
Newton's laws of motion [54]) is computed as follows:

$$
D^{\prime}[i]=\frac{F[i]}{M[i]}
$$

where $F[i]$ is an elastostatic force and is computed for every node from the direct force applied on it and the force it receives from the neighboring nodes. As the Young's modulus accounts for the mass, the value of $M[i]$ is set to 1 . The elastostatic force is computed using the following physical principle. A given amount of direct force that is applied on any node on the surface is transferred in part to the neighbors of that node in a particular ratio given by a transfer matrix. This transfer of force is caused by the elastic interaction, which causes the neighboring nodes to exhibit displacement. The transfer matrix contains the ratio with which the direct force is transferred between nodes. This principle is mathematically represented by the Green's formulation.

Let $f[j]$ be the direct force applied on node $j$ and $T[j \rightarrow i]$ be the transfer matrix element of the Green's formulation that represents the transfer of force from node $j$ to $i$. The resulting elastostatic force $F[i]$ can be written as

$$
F[i]=\sum_{j=0}^{N} f[j] \times T[j \rightarrow i]
$$

where $N$ is the total number of nodes. Some of the key properties of the transfer matrix are as follows. The dynamics of airflow inside lungs follow fluid dynamic properties, which eliminate the contact force on each lung node. Such fluid flow properties allow the air to flow into regions of lower resistance. The variation in regional resistance to expansion is caused by the heterogeneous expansion of alveoli. While the transfer matrix is thus not symmetric as previously used in traditional methods, the summation of its elements for a given value of $j$ is also set to 1 as follows:

$$
\sum_{j=0}^{N} T[j \rightarrow i]=1 .
$$

Using (3), (2) can be expanded as

$$
F[i]=f[i]+\sum_{j=0, j \neq i}^{N} f[j] T[j \rightarrow i]-\sum_{j=0, j \neq i}^{N} f[i] T[i \rightarrow j] .
$$

From (4), it can be seen that the elastostatic force is a summation of the force directly applied on a node $i$ and the force received from its neighboring nodes, subtracted by the direct force of $i$ transferred to its neighbors. Thus, for a known transfer matrix and applied force, the displacement can be computed using (4). Equation (4) describes the force distribution that occurs in an elastic displacement of nodes. However, since the transfer matrix is not known, we consider a localized estimation of the transfer matrix elements, which is based on an elastic equilibrium caused by the laws of conservation of energy. Let $T_{e}[j \rightarrow i]$ be the estimated transfer matrix element whose estimation will be discussed in Section III-G. The elastostatic force applied on surface nodes under elastic equilibrium can be represented as

$$
F[i]=F[i] \times T_{e}[i \rightarrow i]+\sum_{j=0, j \neq i}^{N} F[j] \times T_{e}[j \rightarrow i] .
$$

Equation (5) is an iterative equation, which represents the local balance among the forces acting on each node of an elastic surface, which in our case is a mesh lung model. In order to solve this equation an iterative solution is required. The initial value $F[i]$ on the right-hand side of (5) is substituted for $f[i]$. Equation (5) is now rewritten as

$$
F[i]=f[i] \times T_{e}[i \rightarrow i]+\sum_{j=0, j \neq i}^{N} F[j] T_{e}[j \rightarrow i] .
$$

Equation (6) is still an iterative equation as every neighbor of node $i$ experiences a similar force distribution process. Additionally, every node receives an elastostatic force based on the elastic interaction among the nodes. Since in the real world, the lung tissues are thin, no vibrations are seen during lung deformations, and the elastostatic force is assumed to reach its equilibrium in a negligible amount of time. Also, the lung deformations are observed to be linear with an increase in volume (as explained in Section III-B). For a known value of the estimated transfer matrix and applied force, the elastostatic force can be derived. The values of the transfer matrix $T$ can be computed by equating the right-hand side of (2) and (5), and by considering the magnitude vectors $f$ and $F$ as diagonal matrices. The elements of the transfer matrix $T$, can thus, be written as

$$
T[j \rightarrow i]=\left(\frac{F[j]}{f[j]}\right) \times T_{e}[j \rightarrow i] .
$$

\section{G. Iterative Solutions for Displacement Computation}

We now proceed on solving (6) to estimate the elastostatic force for an initial estimation of the applied force. An iterative method to compute the elastostatic force starts with an initial estimation of the transfer matrix. Using the estimation of the transfer matrix and the applied force, we compute the elastostatic force. The estimated transfer matrix $\left(T_{e}\right)$ is computed for every node pair $j$ and $i$. To estimate the transfer matrix, a regularly connected mesh, as observed in high-resolution 3-D models, is considered. Let us consider an accumulator matrix TP used by the transfer matrix for storing intermediate computations. The transfer matrix from every node to every other node is initially set to 0 , and then, computed as follows. The transfer matrix from a node $j$ to its immediate neighbor $i$ is first computed by dividing the Young modulus of $i$ by the summation of Young's modulus of nodes in the immediate neighborhood of $j$, and is given as

$$
\begin{aligned}
& T_{e}[j \rightarrow i]=T P[j \rightarrow i]+\left(\frac{S_{i}}{\sum_{l=0}^{\operatorname{cliqueof}(j)}\left(S_{l} \times\left(\frac{1}{\operatorname{Dist}(l, j)}\right)\right)}\right) \\
& \times \frac{1}{\operatorname{Dist}(j, i)} \\
& T P[j \rightarrow i]=T_{e}[j \rightarrow i]
\end{aligned}
$$




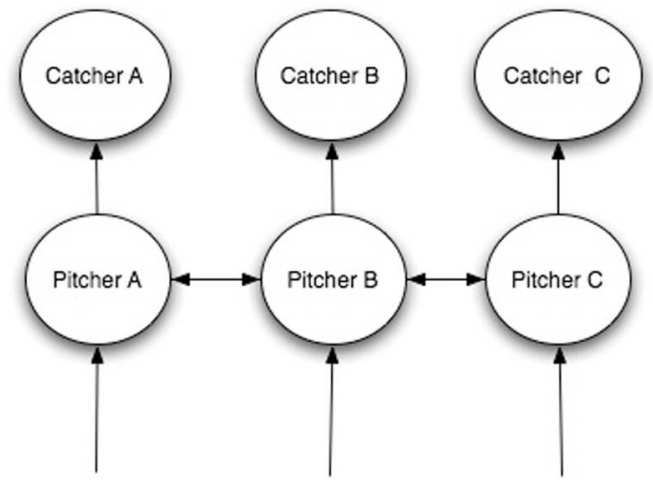

Fig. 6. Schematic representation of the accumulators used for the iterative solution.

where $S_{i}$ is the inverse of the Young modulus of the $i$ th node if $i$ is a mass node. With respect to the spring node, the value of $S_{i}$ is the Young modulus. The $\operatorname{Dist}(i, j)$ is a function that represents the Euclidean distance between the $i$ th and $j$ th node. The minimum value of $\operatorname{Dist}(i, j)$ is set to be $l$. The cliqueof $(j)$ represents the immediate neighboring nodes of $j$. The transfer matrix is then propagated from $j$ through $i$ to other nodes until the contribution reaches 0 in the following manner. The transfer matrix from node $j$ to a node $k$, which is an immediate neighbor of node $i$ and not an immediate neighbor to node $j$ is given as

$$
\begin{aligned}
T_{e}[j \rightarrow k] & =T P[j \rightarrow k]+T_{e}[j \rightarrow i] \times T_{e}[i \rightarrow k] \\
T P[j \rightarrow k] & =T_{e}[j \rightarrow k] .
\end{aligned}
$$

The value of $T_{e}[i \rightarrow k]$ is computed using (8). Equation (10) is used for computing the transfer matrix for all nodes reachable from the node $j$ along a forward path. Equations (8)-(11) will always converge because the transfer matrix between any two nodes is always less than 1 . The result of (8)-(11) gives an initial estimation of the transfer matrix.

To compute the elastostatic force, it is first initialized to the applied force. The force applied on a spring node is initially set to 0 since these nodes are used for damping the flow of force between two mass nodes. Two sets of accumulators for each node are introduced at each iteration time step $t$, which are named Catcher ${ }^{t}$ and Pitcher ${ }^{t}$, respectively. While the former provides the final value of the force that causes the displacement for a node, the latter indicates the force, which needs to be distributed to its neighbors if it is greater than 0 . A schematic representation of the applied force and accumulators are shown in Fig. 6. During a sequence of iterations denoted by $t$, a node $i$ of maximum Pitcher ${ }^{t-1}$ is chosen and the force in that accumulator is distributed to other nodes as follows

$$
\text { Pitcher }^{t}[j]=\text { Pitcher }^{t-1}[j]+\text { Pitcher }^{t-1}[i] \times T_{e}[i \rightarrow j]
$$

$$
\text { Catcher }^{t}[i]=\text { Catcher }^{t-1}[i]+\text { Pitcher }^{t-1}[i] \times T_{e}[i \rightarrow i]
$$

$$
\text { Pitcher }^{t-1}[i]=0 \text {. }
$$

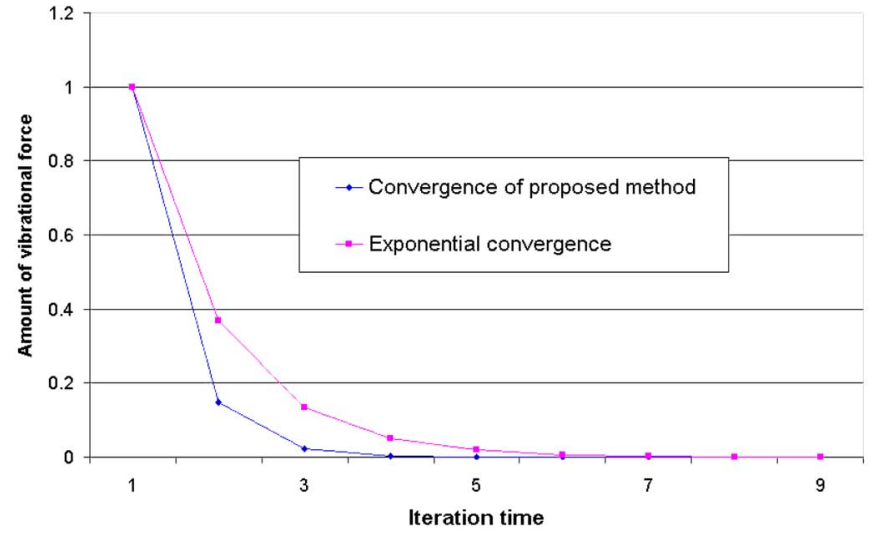

Fig. 7. Convergence of the proposed method as compared to the exponential convergence of the mass-spring-damper model.

It can be seen that the Pitcher ${ }^{t}$ accumulator reaches zero for all the nodes for higher values of $t$. Each node $i$ is either a mass node or a spring node. The elastostatic force $F[i]$ of a mass node $i$ in the model is given by the Catcher $^{t}[i]$ accumulator. The Catcher $^{t}[i]$ for a spring node $i$ is set to be the average of its two mass nodes for deformation purposes. The aforementioned equations are repeated in the same order until the Pitcher accumulator of all the nodes becomes 0 . Thus, the iterative solution can compute the equilibrium displacement of each of the model nodes for a given applied force. This completes the computation of the elastostatic force that can be now used for animation of the lung model. Before we proceed with it, first we have to verify the validity of the iterative solution. Fig. 7 shows the convergence of the proposed method as compared to the exponential convergence of the mass-spring-damper model. The convergence of the proposed method is superior as compared to the mass-spring-damper model.

\section{VALIDATION PROCEDURE}

The validation procedure first focuses on the accuracy of the proposed mathematical model. It is then followed by the validation of lung deformations.

\section{A. Validation of the Proposed Mathematical Model}

The role played by physically based deformations for the deformation of both homogenous and heterogenous elastic models was discussed in Sections II-A and B. In order to account for variations in the lung's elastic property, the proposed mathematical model needs to be validated for both homogenous and heterogenous elastic models. For validating the mathematical model, the mass-spring-damper model [11] is chosen and simulated for both homogenous and heterogenous models using the Kineticskit source code's graphical interface [55]. The same graphical interface was also used to simulate and compare the deformation method we proposed.

The mesh of the mass-spring-damper model was considered to have homogenous elastic properties. A force pattern similar to the expansion of lung models (bending force) was applied on each node of the mesh. The original mesh and the deformed 


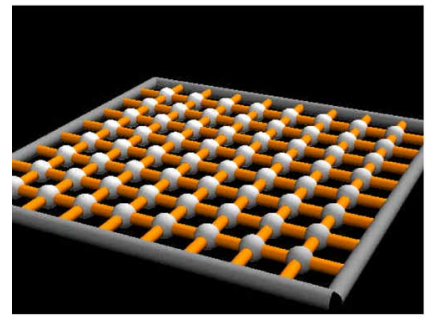

(a)

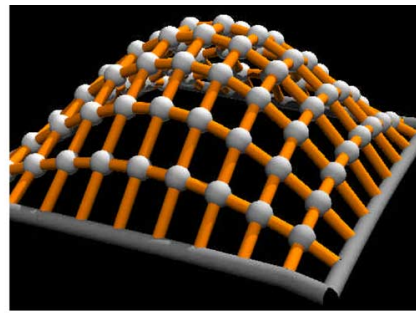

(b)

Fig. 8. (a) Regular planar mesh of isotropic Young's modulus. (b) Deformed shape of the regular mesh using the proposed method of deformation.

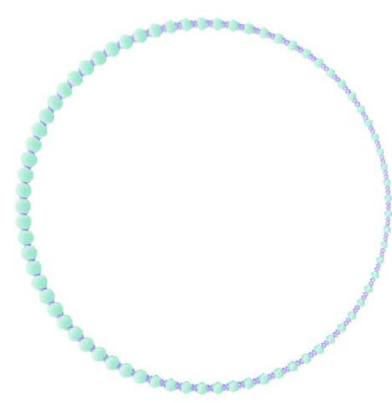

(a)

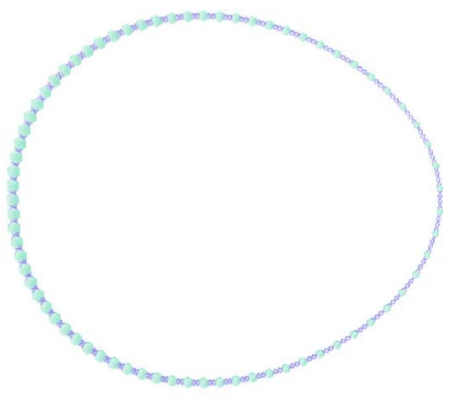

(b)
Fig. 9. (a) Regular 2-D circularly connected mesh of nodes (spheres). The higher the radius of the node the higher is the Young's modulus. (b) Deformed 2-D circularly connected mesh. A balloon-like expansion can be seen in the deformed state.

mesh using the proposed method are shown in Fig. 8(a) and (b). The difference in the displacement using the two methods is less than $0.1 \%$ RMS.

Fig. 9(a) shows a 2-D circular mesh with nodes (spheres) of variable size. The higher the radius of the node, the higher is their Young's modulus. This 2-D mesh is deformed by applying a unit force on all the nodes along their radius. Using the proposed method of computation, the 2-D deformed mesh is as shown in Fig. 9(b). It can be seen that the radial displacement of nodes with higher Young's modulus is less than $0.1 \%$ RMS, which illustrates the absence of fixed contact force on the nodes.

\section{B. Validation of 3-D Lung Dynamics}

The validation of 3-D lung deformations is done by: 1) validating the linearity in the direction of 3-D lung deformation for every node using the proposed method; 2) validating the deformation; 3) estimating the applied force $f$ from the 4-D HRCT dataset coupled with (6), and 4) and verifying that it correlates with the gradient of gravity caused by the orientation of the human subject. The relation between the airflow and the gradient of gravity has been previously quantified.

1) Validation of the Displacement Direction: We compute the directions of the displacement of the model nodes using 3-D clinical data analysis while we compute the magnitude of applied force using (6). A sequence of three 3-D lung models obtained from a normal human lung at $5 \%, 40 \%$, and $75 \%$ tidal volume at supine position were considered for analysis. The supine position imposes no downward movement of the lung tissue at the apex and no forward movement at the posterior part of the lung. Thus, the apex is chosen as the origin for the $Z$-axis, the posterior part of the lung as the origin for the $Y$-axis, and the heart as the origin for the $X$-axis. The surface node of these 3-D models at various tidal volumes needs to be put in correspondence in order to compute the displacement of nodes. Although various correspondences corresponding to different directions of deformation could be established, one direction for each node will yield a linear displacement when the lungs are expanding from $5 \%$ to $40 \%$ and from $5 \%$ to $75 \%$ tidal volume. The correspondences are established by projecting a ray from a node of the $3-\mathrm{D}$ model at $5 \%$ tidal volume in a specific direction, and performing a ray-triangle intersection analysis of that ray with the lungs at a higher tidal volume. The specific direction is estimated as follows. Let $\min$ and max be two vectors that represent the bounding coordinates of the lungs at $5 \%$ tidal volume. Let $p_{i}$ be the position of node $i$ at $5 \%$ tidal volume and $d_{i}$ be a vector that represents the estimated direction of node $i$. Under the hypothesis that the direction of displacement is constant, the components of $d_{i}$ may be simply modeled as first-order polynomials given by

$$
\begin{aligned}
& d_{i} X=c_{1} \times\left(\frac{p_{i} X-\min X}{\max X-\min X}\right)^{c_{2}} \\
& d_{i} Y=c_{3} \times\left(\frac{p_{i} Y-\min Y}{\max Y-\min Y}\right)^{c_{4}} \\
& d_{i} Z=c_{5} \times\left(\frac{p_{i} Z-\min Z}{\max Z-\min Z}\right)^{c_{6}}
\end{aligned}
$$

where $\mathrm{c}_{1}, \mathrm{c}_{2}, \mathrm{c}_{3}, \mathrm{c}_{4}, \mathrm{c}_{5}$, and $\mathrm{c}_{6}$ are constants.

The choice of this first-order polynomials are now validated by ensuring that their use yields the correct lung deformation given by medical data within $1 \%$ rms error. The required values for the constants must allow the displacement's magnitude of every node to be linear with an increase in volume as previously observed in [47]. The constants were estimated by an exhaustive searching approach to choose their values in order to yield a minimum RMS deformation error. The searching approach may be described as follows. The initial value of the constants was set to be 0.01 . We then computed different combinations of values for the constants, with the difference between two consecutive values of a constant set to 0.01 . For each combination, we computed the displacement of surface nodes.

The values of the computed constants that provided an RMS error of less than $1 \%$ are given in Table I. Results of the corresponding deformations obtained are now detailed. Fig. 10(a) and (b) shows the side view of the left and right lungs at $40 \%$ tidal volume (gray model) overlapped with the corresponding left and right lungs projected along the directions computed from (15)-(17) when expanding from 5\% to $40 \%$ tidal volume (bright model). The overlap in each of these cases was computed using Geomagic Software to be within 1\% RMS (2 mm) surface distance error. Such results support the hypothesis that the direction of displacement of the surface nodes of a 3-D lung 
TABLE I

TABulation of CONSTANTS ESTIMATED From A NoRMAL HuMAN SubJECT

\begin{tabular}{|l|l|l|l|l|l|l|l|l|l|}
\hline Lung & C1 & C2 & C3 & C4 & C5 & C6 & C7 & C8 \\
\hline Left & 0.09 & 0.23 & 0.4 & 0.5 & 0.1 & 2.3 & 0.01 & 0.95 & 0.07 \\
\hline Right & 0.09 & 0.22 & 0.39 & 0.51 & 0.11 & 2.2 & 0.01 & 0.96 & 0.05 \\
\hline
\end{tabular}

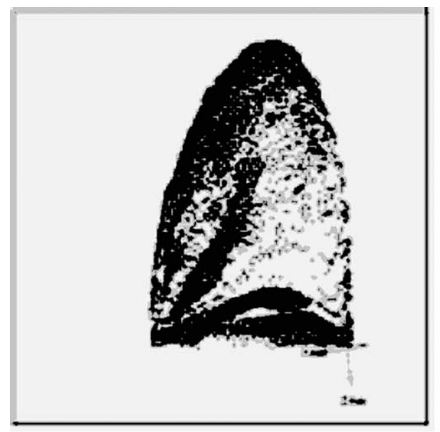

(a)

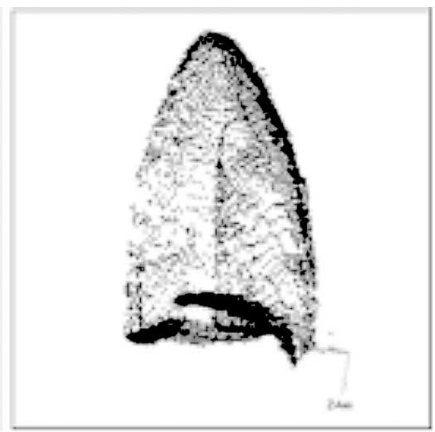

(b)

Fig. 10. (a) Side view of a 3-D left lung model at $40 \%$ tidal volume (gray color) and a 3-D left lung model obtained by deforming the left lung at 5\% tidal volume to $40 \%$ tidal volume (bright color). (b) Side view of a 3-D right lung model at $40 \%$ tidal volume (gray color) and a 3-D right lung model obtained by deforming the left lung at $5 \%$ tidal volume to $100 \%$ tidal volume using the same direction of displacement (bright color).

model under normal breathing conditions can be modeled as a constant.

2) Validation of 3-D Lung Deformations: The validation of 3-D lung deformations is performed in two approaches, which are: 1) comparing the shape of simulated lung shape with subject lung shape (morphometric validation) and 2) comparing the positions of biologically relevant landmarks in simulated lung shape and subject data.

The ability to accurately simulate the 3 -D lung shape at $100 \%$ tidal volume validates the proposed method of lung deformation. Fig. 11(a) and (b) shows a side view of the left and right lungs at $100 \%$ tidal volume (gray model) overlapped with the left and right lungs projected along the same directions computed from (15)-(17) when the lung is expanding from $5 \%$ to $100 \%$ tidal volume. The magnitude of the displacement in this case was computed by linearly scaling the nodal displacement computed for a deformation from 5\% to $40 \%$ tidal volume.

Biologically relevant landmarks were chosen on the 3-D models of human subject data at 5\%, and the same landmarks are also chosen on the 3-D models of human subject data at $100 \%$ tidal volume. The lung model at $5 \%$ tidal volume, is then, deformed using the proposed method The new lanrmark positions matched with the landmark positions marked on the human subject data at $100 \%$ tidal volume with less than $2 \mathrm{~mm}$ departure. Fig. 12(a) and (b) shows the simulated landmarks (gray) and landmarks chosen on the human subject data at $100 \%$ tidal capacity (bright).

3) Validation of the Applied Force: The magnitude of force applied on each node of the dataset, is then, computed using (6) with the displacement estimated from the aforementioned

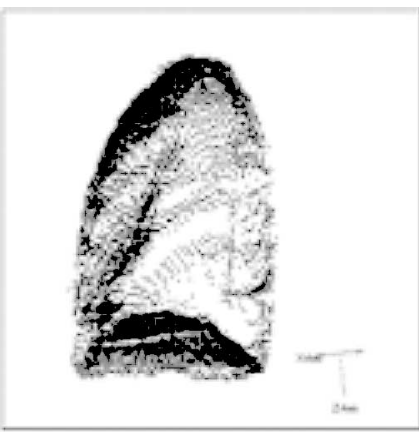

(a)

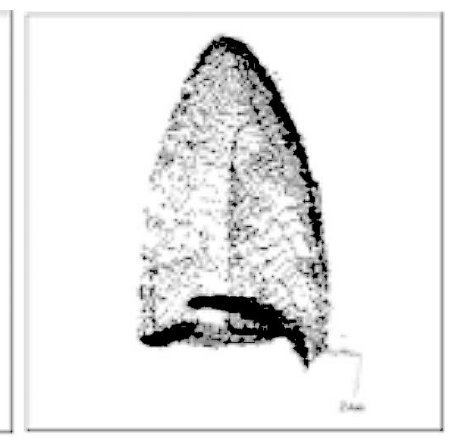

(b)
Fig. 11. (a) Side view of a 3-D right lung model at $100 \%$ tidal volume (gray color) and a 3-D left lung model obtained by deforming the left lung at 5\% tidal volume to $100 \%$ tidal volume (bright color). (b) Side view of a 3-D right lung model at $100 \%$ tidal volume (gray color) and a 3-D right lung model obtained by deforming the right lung at $5 \%$ tidal volume to $100 \%$ tidal volume (bright points) using the same direction of displacement used for Fig. 10. The magnitude of the displacement in this case is computed by linearly scaling the nodal displacement computed for a deformation from $5 \%$ to $40 \%$ tidal volume.

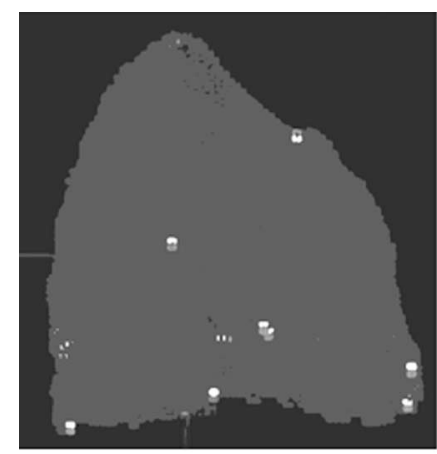

(a)

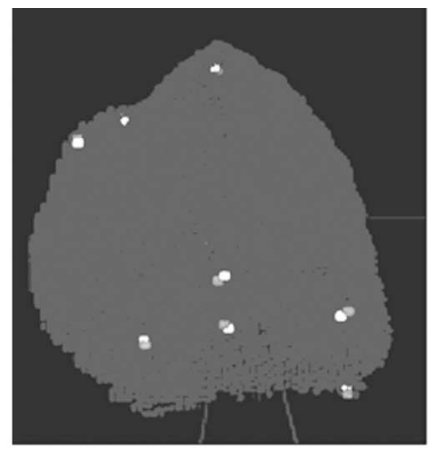

(b)
Fig. 12. (a) Side view of a $3-\mathrm{D}$ right lung model at $100 \%$ tidal volume in which the landmarks chosen on the subject data (gray color) and the simulated landmarks (bright color) are shown. (b) Side view of a 3-D right lung model at $100 \%$ tidal volume in which the landmarks chosen on the subject data (gray color) and the simulated landmarks (bright color) are shown.

steps and the transfer function estimated using (8) and (9). The next step is to compute the magnitude of the applied force as a function of node position. The applied force on a node $i$ is first normalized to fit within a range of $0-1$, and then, approximated using the function

$$
\begin{aligned}
f[i]=c_{7} & \times\left(\frac{p_{i} X-\min X}{\max X-\min X}\right)+c_{8} \\
& \times\left(\frac{p_{i} Y-\min Y}{\max Y-\min Y}\right)+c_{9} \times\left(\frac{p_{i} Z-\min Z}{\max Z-\min Z}\right)
\end{aligned}
$$




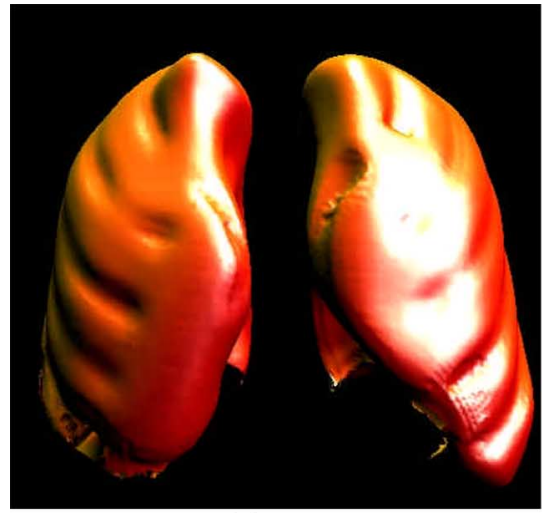

(a)

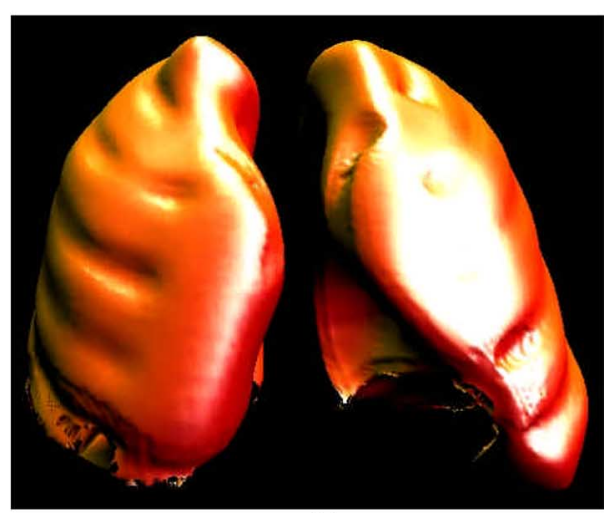

(b)

Fig. 13. Deformation of a high-resolution lung models obtained from a normal human subject, using the proposed approach. (a) Lung at residual volume (i.e., before inhalation). (b) Deformed lung at the end of inhalation.

where $c_{7}, c_{8}$, and $c_{9}$ are constants, which are estimated by an exhaustive search approach with the constraint that the aforementioned function best fits the applied force computed from medical data. Using (16), the applied force of the given 4-D lung dataset is modeled to satisfy an RMS error of less than $1 \%$. The values of these constants are also tabulated in Table I, which can be used for obtaining a physically valid 3-D lung deformation. It can be observed that the values of $c_{7}$ andc ${ }_{9}$ are very less as compared to the value of $c_{8}$, which shows the effect of the gravity (along the $Y$-axis) on the applied force. It must also be noted that the values of these constants can be used in simulating 3-D lung deformations in supine orientation for any 3-D static lung model such as the visible human dataset. Such a simulation would yield a deformation similar to the 4-D HRCT dataset. Datasets are now being collected on which a statistical analysis of these constants will be conducted in order to establish their range of variability across a given population, which in a first step will be normal subjects.

\section{REAL-TIME DEFORMATIONS}

We have discussed a physics- and physiology-based method for modeling 3-D lung deformations. Using this method, we can now animate various 3-D lung models in two approaches. In the first approach, for real-time deformation, we make use of the Graphics Processing Unit's vertex shaders to compute the lung deformation. In addition, we also make use of the spherical harmonic ( $\mathrm{SH}$ ) transformations to reduce the computational complexity of the matrix-vector product (of the transfer function and the applied force). Such an approach allows us to use the applied force approximated for different subject orientation, in order to obtain the real-time lung deformations for subject at different orientations. This implementation, which is further detailed in [56], is then integrated into the AR environment discussed in Section I. This implementation, has a per-vertex computation of approximately 16 multiplications and 18 additions, which is lower as compared to the per-vertex computation of (2).

In the second approach, we discuss a method for deforming 3-D lung models for static subject orientations. When compared to the first approach, the second approach would further reduce
TABLE II

IMPLEMENTATION SYSTEM INFORMATION

\begin{tabular}{|l|l|}
\hline \multicolumn{2}{|l|}{ Implementation system information } \\
\hline CPU & Athlon 2800 \\
\hline GPU & GeForce4 Ti 5600 \\
\hline Shaders & NVIDIA CG 1.1 \\
\hline & \\
\hline Op. System & Linux Redhat 8.0 \\
\hline
\end{tabular}

the per-vertex computation to three additions, which improves the scalability of the simulation and visualization environment. In this case, the displacement of each node is precomputed for a unit-applied force and for a given orientation of the patient. The precomputed displacement is used for deforming 3-D lung models in real time across the whole breathing cycle using the linearity in displacement from lower to higher tidal volumes. Such an approach reduces the run-time complexity of deforming a 3-D lung model to three additions per node. We also use state-ofthe-art graphics processing units in order to avoid any rendering delays. Fig. 12(a) shows the initial shape of a high-resolution 3D lung model obtained from the 3-D HRCT datasets of a normal human subject other than the one used in the analysis done in Section IV-B. The unit increase in volume was set as the ratio of the tidal volume of human lungs (i.e, $500 \mathrm{~mL}$ ), to the product of the deformation steps per second (i.e, $66.66 \mathrm{steps} / \mathrm{s}$ ) and the ventilation rate of inhalation or exhalation (normally $5 \mathrm{~s}$ ). For this increase in volume, the applied force for the lungs in the supine position was computed using (18). The deformed shape caused by inhalation is as shown in Fig. 13(b). The run-time deformation takes $O(n)$ operations where $n$ is the number of nodes. The implementation system details are explained in Table II.

\section{DISCUSSION}

Through our research, we aim to extend the thoracic simulation paradigm to include real-time visualization of 3-D lung dynamics. This is achieved using the real-time precomputationbased approach for lung dynamics discussed in this paper, which closely models the change in 3-D lung shape. Such an approach coupled with the PV relation (discussed in Sections III-A and III-B) allows us to simulate normal 3-D lung deformations. 
Our method focuses on physics- and physiology-based subject-specific 3-D deformable lung models. The 3-D deformable models being generated from 4-D HRCT imaging show the effective role that such advanced imaging systems can play in deciding clinical interventions for a wide range of disease states. Additionally, the 3-D deformable lung models also form a feasible and cost-effective replacement for 4-D HRCT imaging under dynamics conditions. Coupled with the AR-based visualization, such deformable models facilitate the prototype to have user-specific views of the subject-specific lungs.

The proposed method takes into account the deformation constraints imposed by the diaphragm and rib cage (through the PV curve) on the lung's air volume and the regional alveolar expandability on the regional lung shape. The aforementioned physiological components can be individually varied in order to obtain physically realistic variations of 3-D lung deformation. For instance, pneumothorax, a pathophysical state in which the change in 3-D lung deformations is caused by an external wound, is demonstrated using the proposed 3-D lung deformations by varying the PV relation and static lung shape [57]. Similarly, tumor-influenced lung dynamics, in which a change in 3-D lung deformations is caused by the presence of lung tumor, is demonstrated using the proposed 3-D lung deformations by varying the regional alveolar expandability [58].

The choice of a single compartmental model enables visualization of high-resolution 3-D lung deformations. The accuracy in the usage of a single compartmental model has been validated by some of the peers. The validation, however, needs to be performed across a wide range of human subjects of various age and race. Additionally, the applicability of a singlecompartmental model to simulate diseased lung dynamics needs to be further validated. The simulated lung dynamics needs to be compared with 3-D HRCT images of normal lung subjects with different breathing conditions.

The usage of a physically based deformation approach for lung deformations allows us to model lung deformations with variations in physics-based parameters. The usage of the regional alveolar expandability as one of the parameters allows the proposed method to account for the physiology of normal human subjects. The method can be extended by analyzing the regional alveolar expandability for human subjects across a wide range of age and race. An inverse analysis of the proposed method can also be used to estimate the alveolar expandability [59]. Such an analysis of physiology would facilitate modeling the 3-D lung dynamics for a wide range of human population. Additionally, the variations in the air constituents also need to be included in the current method. One may note that the variations in the air constituents can lead to changes in the alveolar blood pressure, which subsequently alters the alveolar expandability. The proposed method can be expanded in order to address this aspect of the lung physiology.

The validation of the proposed method of deformation was discussed in Section IV-A and the simulated lung deformation in Section IV-B. Through this validation, we demonstrated obtaining physically and physiologically based lung deformations. Additional validations can be done using HRCT data obtained from a higher number of normal and diseased human subjects under different breathing conditions. The validation can also be done by generating physically based deformable lung models using our human subject data as explained by the peers and comparing it with the proposed simulation method. The results of such a validation would discuss the feasibility of the proposed method in different breathing conditions of the human subject and will be discussed in the future.

From the clinical usage perspective, the proposed method can be considered for training and planning under three conditions, which are: 1) without any invasive intervention; 2) with minimal invasive intervention; and 3) with thorough invasive intervention.

1) Without any invasive intervention: Such clinical scenarios will include investigating a patient's general breathing patterns and discomfort (e.g., dyspnea). For this case, the proposed method allows the user to simulate and view subject-specific breathing as 3-D lung surface deformations under different physical conditions and orientations of the patient.

2) With minimal invasive intervention: Such interventions will include procedures such as intubation, endoscope, needle insertion, and radiation oncology treatments. The proposed method would be an effective tool since it can show the position of the minimally invasive tools as well as the breathing changes (external surface shape changes) that are caused by the subjective discomfort and the effect of the clinical intervention. The real-time capability of the proposed method may further extend the utility of the clinical applications for real-time clinical guidance.

3) With thorough invasive intervention: Such interventions will include preplanned procedures such as lung transplants and lung volume reduction. Under such interventions, the proposed method would be an effective tool for visualizing preoperative conditions and postoperative prognosis for the patient. Emergency events, which occur along with lung transplants and volume reduction surgery, are discussed in [51]. Simulating intraoperative conditions would heavily rely on the biomathematical interactive 3D models that can accurately account for user-induced variations in the subject's anatomy.

\section{CONCLUSION}

We demonstrated the precomputation of physically based lung deformations and the deformations of a high-resolution lung model. The deformation being independent of the absolute assignment of the initial Young modulus of each node facilitates easier creation of physically based deformation. The precomputation approach also provides real-time deformations, which are highly suitable for AR environments. The proposed method was validated by: 1) resimulating the lung deformation and comparing it with the actual patient data and 2) estimating the applied force extracted from the 4-D HRCT data. For additional validation, we are currently investigating the process of analyzing more patient data using invasive methods. The results of this study will be discussed in future work. 


\section{ACKNOWLEDGMENT}

The authors thank Dr. E. A. Hoffman and Dr. W. P. Segars for providing clinical data for modeling lung deformations; Dr. S. N. Pattanaik (School of Computer Science, University of Central Florida) and Dr. T. Santhanam (Department of Physics, St. Louis University) for their comments; and C. M. Fidopiastis (Institute of Simulation and Training, University of Central Florida) Dr. F. G. Hamza-lup (Department of Computer Science, Armstrong Atlantic State University), and C. Balgeman (School of Arts, University of Central Florida) for their constructive comments and editing.

\section{REFERENCES}

[1] M. J. Ackerman, "The visible human project," in Proc. IEEE, vol. 86, no. 3, pp. 504-511, 1991.

[2] B. Q. Tran, J. K. Tajik, R. A. Chiplunkar, and E. A. Hoffman, "Lung volume control for quantitative X-ray CT," J. Biomed. Eng., vol. 24, p. s-66, 1996.

[3] J. P. Rolland, L. Davis, and F. Hamza-Lup, "Development of a training tool for endotracheal intubation: Distributed augmented reality," Med. Meets Virtual Reality (MMVR), vol. 11, pp. 288-294, 2003.

[4] D. Terzopolous, J. Platt, A. Barr, and K. Fleisch, "Elastically deformable models," ACM Siggraph, vol. 21, no. 4, pp. 205-214, 1987.

[5] S. M. Platt and N. I. Badler, "Animating facial expressions," in Proc. 8th Annu. Conf. Comput. Graph. Interact. Tech., 1981, pp. 245252.

[6] M. Alexa, "Recent advances in mesh morphing," Comput. Graph. Forum, vol. 21, no. 2, pp. 173-196, 2002.

[7] X. Wang and V. Devarajan, "2D structured mass-spring system parameter optimization based on axisymmetric bending for rigid cloth simulation," in Proc. ACM SIGGRAPH Int. Conf. Vir. Reality Continuum Appl., New York, NY: ACM, 2004, pp. 317-323.

[8] Y. Zhang, E. C. Prakash, and E. Sung, "Real-time physically-based facial expression animation using mass-spring system," in Proc. Comput. Graph. Int., IEEE Press, Piscataway, NJ, 2001, pp. 347-350.

[9] A. Aubel and D. Thalmann, "Realistic deformation of human body shapes," in Proc. Comput. Animations Soc. Agents, 2000, pp. 125135 .

[10] F. Boux deCasson and C. Laugier, "Modeling the dynamics of a human liver for a minimally invasive surgery simulator," in Proc. MICCAI, Cambridge, U.K., 1999, pp. 1156-1165.

[11] R. D. Blevins, Flow-Induced Vibration. New York: Van Nostrand, 1990.

[12] M. Desbrun, P. Schroder, and A. Barr, "Interactive animation of structured deformable objects," in Proc. Graph. Interface 1999, pp. 1-8.

[13] M. Bro-Nielsen, "Finite element modeling in medical VR," J. IEEE, vol. 86, no. 3, pp. 490-503, Mar. 1998.

[14] D. L. James and G. K. Pai, "Multiresolution green's function methods for interactive simulation of elastostatic models," ACM Trans. Graph., vol. 22, no. 1, pp. 47-82, 2003.

[15] Y. Zhuang, "Real-time simulation of physically-realistic global deformations," Ph.D. dissertation, Dept. Elect. Eng. Comput. Sci., UC, Berkeley, Fall, 2000.

[16] S. Cotin, H. Delingette, and N. Ayache, "Real-time elastic deformations of soft tissues for surgery simulation," IEEE Vis. Comput. Graph., vol. 5, no. 1, pp. 62-73, Mar. 1999.

[17] R. E. White, An Introduction to Finite Element Methods. Raleigh, NC: Wiley, 1991.

[18] T. M. MacRobert and T. Murray, "Spherical harmonics an elementary treatise on harmonic functions with applications," in International Series of Monographs in Pure and Applied Mathematics. New York: Oxford, 1967.

[19] I. Stakgold, "Green's functions and boundary value problems," in Mathematical Physics. Hoboken, NJ: Wiley, 1979.

[20] D. L. James and G. K. Pai, "ARTDEFO: Accurate real-time deformable objects," in Proc. Annu. Conf. Comput. Graph. Interact. Tech., 1999, vol. 33, pp. 65-72.

[21] G. Nakamura and K. Tanuma, "A formula for the fundamental solution of anisotropic elasticity," Quaterly J. Appl. Math., vol. 50, pp. 179-193, 1996.
[22] T. C. T. Ting and V.-G. Lee, "The three-dimensional elastostatic Green's functions for general anisotropic linear elastic solids," Quaterly J. Appl. Math., vol. 50, pp. 407-426, 1996.

[23] A. L. Levin, "Christoffel functions and orthogonal polynomials for exponential weights," in Memoirs Amer. Math. Soc., vol. 535, Providence, RI, 1944.

[24] Y. C. Fung, Biomechanics: Mechanics Properties of Living Tissues. New York: Spring, 1993.

[25] A. E. Green and W. Zerna, Theoretical Elasticity. Oxford, U.K.: Clarendon, 1968.

[26] H. Delingette, "Towards realistic soft tissue modeling in medical simulation," in Proc. IEEE Spec. Issue Surg. Simul., 1998, pp. 512-523.

[27] G. Picinbono, H. Delingette, and N. Ayache, "Non-linear and anisotropic elastic soft tissue models for medical simulation," in ICRA, 2001, pp. 305321.

[28] R. Balanuik, "Soft-tissue simulation using LEM-long elements method," Med. Meets Virtual Reality, vol. 85, pp. 38-44, 2002.

[29] R. Balanuik and K. Salisbury, "Soft-tissue simulation using the radial element methods," Int. Symp. Surg. Simul. Soft Tissue Model., vol. 1, pp. 48-58, 2003.

[30] M. Hauth, J. Gross, W. Straber, and G. F. Buess, "Soft-tissue simulation based on measured data," in Proc. Med. Image Comput. Comput. Aided Intervention, 2003, pp. 262-270.

[31] J. R. Barber, "Elasticity," in Solid Mechanics and Its Applications, G. M. L. Gladwell, Ed. Waterloo, Canada: Kluwer, 1992.

[32] W. P. Segars and B. M. W. Tsui, "Study of the efficacy of respiratory gating in myocardial SPECT using the new 4D NCAT phantom," IEEE Trans. Nucl. Sci., vol. 49, no. 3, pp. 675-679, Jun. 2002.

[33] W. P. Segars, D. S. Lalush, and B. M. W. Tsui, "Modeling respiratory mechanics in the MCAT and the spline-based MCAT systems," IEEE Trans. Nucl. Sci., vol. 48, no. 1, pp. 89-97, Feb. 2001.

[34] W. P. Segars, Development of a New Dynamic NURBS-Based CardiacTorso (NCAT) Phantom. Ph.D. dissertation, Univ. of North Carolina at Chapel Hill, 2002.

[35] S. Dawson, "A critical approach to medical simulation," Bulletin Amer. College Surgeons, vol. 87, no. 11, pp. 12-18, 2002.

[36] J. R. Ligas and F. P. J. Primiano, "Respiratory mechanics," in Encyclopedia of Medical Instrumentation, J. G. Webster, Ed. New York: Wiley, 1988, pp. 2550-2573.

[37] E. Promayon, P. Baconnier, and C. Puech, "Physically-based model for simulating the human trunk respiration movements," in Proc. Int. Joint Conf. CVRMED MRCAS, 1997, vol. 1205, pp. 121-129.

[38] D. Decarlo, J. Kaye, D. Metaxas, and J. R. Clarke, "Integrating anatomy and physiology for behavior modeling," in Proc. MMVR, 1995, pp. 81-87.

[39] J. M. Kaye, F. P. J. Primiano, and D. N. Metaxas, "A threedimensional virtual environment for modeling mechanical cardiopulmonary interactions," Med. Image Anal., vol. 2, no. 2, pp. 169-195, 1998.

[40] T. Zhang, H. Keller, R. Jeraj, R. Manon, J. Wesh, R. Patel, J. Fenwick, M. Mehta, T. R. Mackie, and B. Paliwal, "Breathing synchronized delivery-A new technique for raidation treatment of targets with respiratory motion," Int. J. Radiation Oncol. Biol. Phys., vol. 57 no. 2, pp. S185-S186, 2003.

[41] M. H. Tawhai and K. S. Burrowes, "Developing integrative computational models," Anatomical Rec., vol. 275B, pp. 207-218, 2003.

[42] H. Ding, Y. Jiang, M. Furmancyzk, A. Prkewas, and J. M. Reinhardt, "Simulation of human lung respiration using 3-D CFD with macro air sac system," presented at the Int. Western Simul. Conf. Soc. Modeling Simul. New Orleans, LA, 2005.

[43] S. Krishnan, K. C. Beck, J. M. Reinhardt, K. A. Carlson, B. A. Simon, R. K. Albert, and E. A. Hoffman, "Regional lung ventilation from volumetric CT scans using image warping functions," Int. Symp. Biomed. Imag., vol. 1, pp. 792-795, Apr. 2004.

[44] J. E. Cotes, Lung function Assessment and Application in Medicine. Oxford, U.K.: Blackwell Sci., 1993.

[45] A. Santhanam, "Modeling simulation and visualization of realtime 3D lung dynamics," Ph.D. dissertation, Univ. Central Florida, 2006.

[46] A. Santhanam, C. Fidopiastis, and J. P. Rolland, "An adaptive driver and real-time deformation algorithm for visualization of high-density lung models," in Med. Meets Virtual Reality 12, Newport, CA: IOS, 2004, pp. 333-339.

[47] L. Fan, C.-W. Chen, E. A. Hoffman, and J. M. Reinhardt, "Evaluation and application of 3D lung warping and registration model using HRCT 
images," in Proc. SPIE Conf. Med. Imag., San Diego, CA, 2001, pp. 234243.

[48] Analyze Sofware, Mayo-Clinic, AZ, 2003.

[49] E. Weibel, Morphometry of Human Lung. New York: Academic, 1963.

[50] J. B. West, Respiratory Physiology, the Essentials. Philadelphia, PA: Lippincott Williams and Wilkins, 1995.

[51] P. M. A. Calverley, Chronic Obstructuve Pulmonary Disease, P. M. A. Calverley and N. Pride, Eds. London, U.K.: Chapman \& Hall, 1995.

[52] A. Santhanam, C. Fidopiastis, F. Hamza-Lup, J. P. Rolland, and C. Imielinska, "Physically-based deformation of high-resolution 3D lung models for augmented reality based medical visualization," in Proc. Med. Image Comput. and Comput. Aided Intervention, AMIARCS (Lecture Notes on Computer Science), Rennes, St. Malo, 2004, pp. 21-32.

[53] R. De Wilde, J. Clement, J. M. Hellemans, M. Decramer, M. Demedts, R. Boving, and K. P. Van de woestijne, "Model of elasticity of the human lung," J. Appl. Physiol., vol. 81, pp. 254-261, 1981.

[54] A. Witkin, D. Baraff, and M. Kass, "An introduction to physics-based modeling," Siggraph Course, Robot. Ins., Carnegie Mellon Univ., pp. C1C12, 1997.

[55] M. Gritsch, "KineticsKit-A physics based general mass spring system," Open Source Software, 2004.

[56] A. Santhanam, F. Hamza-lup, and J. P. Rolland, "Simulating 3D lung dynamics in a programmable graphics processing unit," IEEE Trans. Inf. Techno. Biomedicine, vol. 11, no. 5, pp. 497-506, 2007.

[57] A. Santhanam, C. Fidopiastis, P. Davenport, J. Anton, and J. P. Rolland, "Real-time simulation of pneumothorax-influenced 3D lung deformations," in Medicine Meets Virtual Reality, San Diego, CA, 2006, pp. 480-485.

[58] A. Santhanam, S. P. Mudur, and J. P. Rolland, "An inverse 3D lung deformation analysis for medical visualization," in Computer Animation and Social Agents. Geneva, Switzerland: Computer Graphics Society, 2006, pp. 229-242.

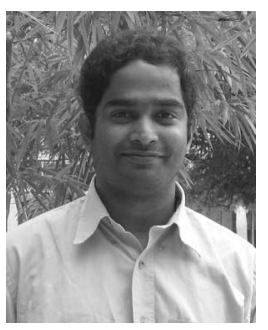

Anand P. Santhanam received the Masters degree in computer science from the University of Texas, Dallas, in 2001, and the Ph.D. degree from the University of Central Florida, Orlando, in 2006.

From April 2000 to January 2001, he was a Software Engineer for Nortel Networks, and from February 2001 to January 2002, he was with Metera Networks. Currently, he is a Joint Postdoctoral Associate in the M.D. Anderson Cancer Center, Orlando, and the College of Optics and Photonics, University of Central Florida. During his Ph.D. research, he was engaged in developing GPU-based algorithms for 3-D lung dynamics. His current research interests include medical image processing, 3-D modeling, rendering, and visualization, and lung tumor dosimetry.

Dr. Santhanam was the recipient of the Link Foundation Fellowship Award for his research during the year 2005 .

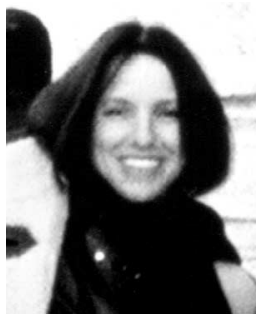

Celina Imielinska received the M.E. degree in electrical engineering from the Politechnika Gdanska, Gdansk, Poland, and the M.S. and Ph.D. degrees in computer science from Rutgers University, New Brunswick, NJ.

Currently, she is the Director of Research for Office of Education and Scholarly Resources, and an Associate Research Scientist in the Department of Medical Informatics, College of Physicians and Surgeons, and the Department of Computer Science, Columbia University, New York. Her current research interests include medical imaging, 3-D visualization, modeling, and simulation.

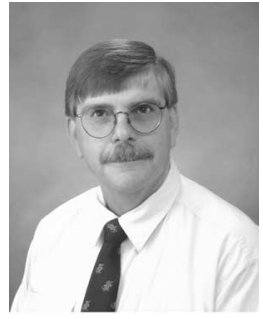

Paul Davenport received the B.A. degree in chemistry from the Greenville College, Greenville, IL, in 1973, and the Ph.D. degree from the Department of Physiology and Biophysics, University of Kentucky, Lexington, in 1980.

He was a Research Associate in the Department of Physiology and Biophysics, University of Texas Medical Branch, Galveston. In 1981, he joined the Department of Physiological Sciences, University of Florida, Gainesville, where he is currently a Professor. His current research interests include the relationship between respiratory mechanics and the neural mechanisms of respiratory sensation.

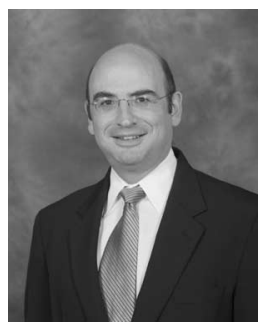

Patrick Kupelian received the M.D. degree from American University of Beirut Medical Center, Beirut, Lebanon.

He completed his Residency in radiation oncology at the University of Texas M.D. Anderson Cancer Center, Houston, and a Fellowship in radiation oncology of genitourinary malignancies at the Cleveland Clinic Foundation, Cleveland, OH. From 1999 to 2002, he was a Clinical Research Director in the Department of Radiation Oncology, Cleveland Clinic Foundation. Thereafter, he joined the M.D. Anderson Cancer Center, Orlando, FL, where he is currently a Director of Research for Radiation Oncology. He has experience with multiple radiation delivery and guidance systems including technologies from Nomos, Varian, BrainLAB, Tomotherapy, and Calypso Medical. He has presented at many congresses, symposia, and specialty meetings in North America and Europe. He is the recipient of research grants from BrainLAB, Tomotherapy Inc, Siemens Medical and Calypso Medical. He is the author or coauthor of more than 100 peer-reviewed publications. His current research interests include hypofractionation, intensitymodulated radiotherapy, and image-guided radiotherapy.

Dr. Kupelian is a member of the American Society for Therapeutic Radiology and Oncology, the European Society for Therapeutic Radiology and Oncology, the American Society of Clinical Oncology, the American Urological Association, and the American Brachytherapy Society.

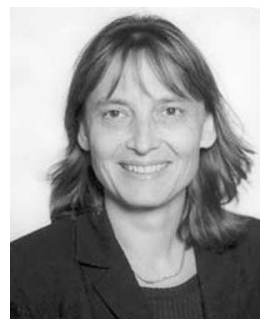

Jannick P. Rolland (M'96) received the Diploma from the Institut D'Optique Graduate School, Paris, France, in 1984, and the Ph.D. degree in optical science from the University of Arizona, Tucson, in 1990.

She is a Professor of Optics as well as a 2007 2012 Chair Professor of the Florida Photonics Center of Excellence, University of Central Florida, Orlando, with joint appointments in Computer Science, Electrical Engineering, and Modeling and Simulation. In 1992, she joined the Research Faculty at the University of North Carolina, Chapel Hill, and during 1992-1996, she became the Head of the Vision Research Group. She is the author or coauthor of more than 170 papers published in journals and conference proceedings and is the author of six book chapters. She is the holder of 13 patents.

Prof. Rolland is a Fellow of the Optical Society of America, and a member of the International Society for Optical Engineering (SPIE) and the SID. She was the recipient of the University of Central Florida 2001 Distinguished Researcher Award and the 2006 Award for Excellence in Teaching. She served on the editorial board of the journal Presence during 1996-2006, and as an Associate Editor of Optical Engineering during 1999-2004. She is a Guest Editor for a special issue of the IEEE JOURNAL OF DISPLAY TECHNOLOGY on medical displays. 